\title{
Sublingual and oral zolpidem for insomnia disorder: a 3-month randomized trial
}

\author{
Laura S. Castro, ${ }^{1}$ iD Leonardo J. Otuyama, ${ }^{1}$ Cristiane Fumo-dos-Santos, ${ }^{1,2}$ Sergio Tufik, ${ }^{1,2}$ \\ Dalva Poyares ${ }^{1,2}$ \\ ${ }^{1}$ Disciplina de Medicina e Biologia do Sono, Departamento de Psicobiologia, Universidade Federal de São Paulo (UNIFESP), São Paulo, SP, \\ Brazil. ${ }^{2}$ Associação Fundo de Incentivo à Pesquisa (AFIP), São Paulo, SP, Brazil.
}

\begin{abstract}
Objective: To evaluate the safety and efficacy of a $5 \mathrm{mg}$ sublingual dose of zolpidem, compared to a $10 \mathrm{mg}$ oral dose, at bedtime and "as needed" following middle-of-the-night awakenings.

Methods: Participants were randomized into an oral group (oral zolpidem $10 \mathrm{mg}$ and sublingual placebo at bedtime and "as-needed") and a sublingual group (oral placebo and sublingual zolpidem $5 \mathrm{mg}$ at bedtime and "as-needed"). Participants underwent medical evaluation, polysomnography, the psychomotor vigilance test, and completed questionnaires.

Results: Of 85 patients, 67 met the criteria for insomnia (48 \pm 10 years; $79 \%$ women) and were randomized. Of these, 46 completed $92 \pm 5$ days of treatment. Mild-to-moderate adverse events were reported by $25 \%$ of the participants, including headache, sleepiness, and dizziness. Both treatments decreased middle-of-the-night awakenings by an average of $-3.1 \pm 2.3$ days/week and increased total sleep time by 1.5 hours. Changes in sleep quality and insomnia severity scores were also favorable and comparable between groups: variation depended on continuation of treatment. Regarding PSG findings, sleep latency decreased more in the sublingual group than the oral group ( $-14 \pm 42$ vs. $10 \pm 29$ min; $p=0.03)$. The psychomotor vigilance test showed minor residual effects 30 minutes after awakening, which reversed after 2 hours.

Conclusions: The safety and efficacy of both zolpidem formulations are comparable. The sublingual $5 \mathrm{mg}$ dose induced sleep more rapidly.

Clinical trial registration: NCT01896336
\end{abstract}

Keywords: Chronic insomnia; polysomnography; psychomotor performance; sleep diaries; clinical effectiveness

\section{Introduction}

Chronic insomnia is one of the most common sleep disorders, affecting one third of the population, and should receive greater recognition. ${ }^{1,2}$ Symptoms are considered clinically relevant if they occur at least three times/week and persist for 3 or more months despite adequate sleep opportunities, leading to sleep dissatisfaction. ${ }^{3}$ Some insomnia patients underestimate total sleep time (TST). It is hypothesized that these individuals have higher cortical arousability and self-reported rates of insomnia, as well as other psychiatric symptoms. Other patients, however, might complain less but present objective sleep disturbance, being at higher risk of metabolic or cardiovascular outcomes. ${ }^{4}$ From a mental health point of view, it is unlikely that insomnia, either chronic or recurrent, is the sole disorder, but is rather part of a complex multimorbidity. Untreated individuals are in a state of chronic sleep deprivation and have an increased risk of accidents, ${ }^{5}$

Correspondence: Laura S. Castro, Rua Napoleão de Barros, 925, $2^{\circ}$ andar, CEP 04024-002, Vila Clementino, São Paulo, SP, Brazil. E-mail: castro@unifesp.br

Submitted Dec 18 2018, accepted Sep 05 2019, Epub Dec 202019. work or social problems, and compromised health and quality of life. ${ }^{1,2}$

According to regulatory agencies, non-benzodiazepine GABA-A receptor agonists are a standard insomnia treatment, and prescription rates are on the rise. ${ }^{1,2,6,7}$ Zolpidem is the most popular non-benzodiazepine GABA-A receptor agonist. In therapeutic dosages, it reduces sleep latency and the frequency of nocturnal awakenings and increases TST and sleep efficiency. Pharmacokinetic parameters could differ for women and older adults, and have been associated with increased residual impairment and adverse events. ${ }^{8}$ These findings have led the U.S. Food and Drug Administration to recommend lower initial dosages for these patients. ${ }^{9}$ Nevertheless, mid- and long-term trials focusing on objective and subjective measures of effectiveness are scarce, ${ }^{10}$ as are descriptions of complementary therapeutic schemes or dosing regimens. ${ }^{11}$

The purpose of our study was to evaluate the mid-term safety and efficacy of a $5 \mathrm{mg}$ sublingual dose of zolpidem 
for reducing sleep latency when administered at bedtime, and "as needed" following middle-of-the-night (MOTN) awakenings, compared to the traditional oral $10 \mathrm{mg}$ dose. To achieve these goals, we designed a three-month randomized, controlled, and double-dummy trial, in which we tested the patients' clinical improvement, reported adverse events, and changes in subjective and objective sleep measures.

\section{Methods}

\section{Sampling and design}

The prospective participants were adults (20-64 years) who reported nocturnal awakenings predominantly before 3:00 a.m. and who had not used psychoactive drugs in the 30 preceding days. Referrals and online subscriptions to the Sleep Institute's website were channels for recruitment. Exclusion criteria were: 1) a history of major neurological, psychiatric, or other medical disorders; 2) a history of substance abuse/dependence; 3 ) a history of daily alcohol use; 4) pregnancy, lactation, or intending to conceive; 5) an apnea and hypopnea index $(\mathrm{AHI})>10$ events/hour; 6) a periodic limb movement index $>15$ events/hour; and 7) blood alterations suggestive of an undiagnosed medical condition.

The protocol included five onsite visits. The first was for screening, when participants gave written informed consent and were examined. The second was for randomization, scheduled the morning after polysomnography (PSG). The third, fourth, and fifth were follow-up visits. Performance was measured with the Psychomotor Vigilance Test (PVT) which was self-applied twice during the day: 30 minutes and 2 hours after awakening. After lab and tests results, the patients were reassessed for continued inclusion.

Eligible participants were assigned (1:1) to receive active doses of zolpidem either as sublingual $5 \mathrm{mg}$ or oral $10 \mathrm{mg}$ tablets. Because sublingual and oral tablets cannot be made to look and taste alike, a double-dummy technique was used with matching placebos of both formulations, which assured participant and investigator blinding. The oral group received kits consisting of oral zolpidem $10 \mathrm{mg}$ and sublingual placebo, which was used at bedtime and "as-needed." The sublingual group received sublingual zolpidem $5 \mathrm{mg}$ and an oral placebo at bedtime, and sublingual zolpidem $5 \mathrm{mg}$ "as-needed." Placebos were indistinguishable in size, shape, color, and taste. Visits 3,4 , and 5 were follow-ups scheduled at weeks 2 , 6 , and 13 , when the kits were dispensed, and unused medication was collected and counted. The bottles provided to participants contained enough tablets for the period between visits. The sponsor provided boxed kits numbered according to a randomization list with sealed envelopes identifying treatment.

\section{Procedures and instruments}

All visits included a medical evaluation, application of questionnaires, and the distribution and collection of sleep diaries. PSG, the PVT, electrocardiogram, and blood testing were performed twice, once before randomization and again during the final visit. Patients were instructed to take a maximum of one tablet from each bottle per night (bedtime and as needed) and record it in their sleep diaries.

Two sleep specialists performed the evaluations. At screening, the participants' history of insomnia, medication use, comorbidities, and related symptomatology were investigated, and adverse events and clinical improvement were assessed during follow-up. Heart rate, blood pressure, and body mass index were assessed in physical examinations. A 12-lead digital electrocardiogram system (Wincardio System, Micromed, Brasília, Brazil) was used. Blood tests included a complete cell count and assessed liver, renal, and thyroid functioning.

\section{Sleep diaries}

Sleep diaries were used to collect data on medication use and sleep schedules. Based on their data, the following were estimated: 1) medication time; 2) bedtime; 3) sleep duration; 4) time of rescue dose ("as-needed" tablet); 5) wake time; 6) rise time; 7) number of nights in which the regular dose was taken ; 8) number of nights in which a rescue dose was taken; 9) number of nights with sleeponset latency $>30$ minutes; 10) number of nights that MOTN awakenings occurred; 11) perceived TST; 12) calculated TST (sleep time minus wake time); 13) sleep efficiency (TST/time-in-bed); 14) alcohol consumption; and 15$)$ mood and sleep quality ratings. ${ }^{12}$

\section{Questionnaires}

The Pittsburgh Sleep Quality Index evaluates sleep schedule, frequency of insomnia/nighttime symptoms, and level of sleep-related problems in the last month. The global score varies from $0-21$, and scores $>5$ indicate poor sleep quality. ${ }^{12,13}$ The Insomnia Severity Index includes seven Likert scales (scored 0-4) that evaluate symptoms and dissatisfaction/distress in the past two weeks. Scores $>7$ (up to 28 ) indicate clinically meaningful insomnia. A reduction of $\geqslant 8$ points suggests at least moderate improvement after treatment. ${ }^{12,14}$ The Karolinska Sleepiness Scale measures alertness in the present moment on a scale from 1-9 ("extremely alert" to "very sleepy/great effort staying awake/fighting sleep"). Electrophysiological signs of sleepiness, such as slow eye movement and alpha-power density, correlate with ratings $\geqslant 7 .{ }^{15}$

\section{Objective sleep, alertness, and performance measures}

PSG was performed with a digital system (EMBLA ${ }^{\circledR}$ S7000, Embla-Systems, Broomfield, CO, USA). Sleep stages, respiratory events, and leg movements were scored according to American Academy of Sleep Medicine criteria. ${ }^{16}$ The selected variables were TST, sleep-onset latency, wake after sleep onset, sleep efficiency, arousal index, percentage of each sleep stage, rapid eye movement (REM) sleep latency, AHI, minimum oxyhemoglobin saturation $\left(\mathrm{SaO}_{2}\right)$, and periodic limb movement index. 
The PVT measures the response to visual stimuli (PVT-192, CWE, Inc., Ardmore, PA, USA). During the 10minute test, patients are instructed to press a button when a light randomly appears on the screen every few seconds. Data are collected on sleepiness, number of errors, hits, lapses, and reaction time. ${ }^{17}$

\section{Outcome measures}

Treatment compliance was defined as the use of bedtime medication at least $75 \%$ of the nights, which was verified using sleep diary data and tablet counts during follow-up visits. Clinicians rated adverse events by their level of association with the study medication (unrelated, possibly related, or probably/certainly related), and decided whether to continue or discontinue treatment. Means and frequencies from the diary data were computed weekly and for the entire treatment period. For delta values, means from the third and final phase of the treatment period (between weeks 6 and 13) were subtracted from baseline means, or from the first and second phase means (i.e., day 1 until week 6). Ordinal responses to the questionnaires were dichotomized to estimate changes in sleep satisfaction. Clinical improvement referred to positive changes in sleep and functioning, as well as symptom remission as rated by physicians during follow-up visits. Partial or no improvement included persistent symptomatology and/or sleeprelated problems.

\section{Statistical analysis}

Sample size estimation (30/group) aimed at finding differences in the proportion of patients with decreased MOTN awakenings and sleep onset latency (20\% in oral $10 \mathrm{mg}$ and $70 \%$ in sublingual $5 \mathrm{mg}$ ) with $80 \%$ power and a $5 \%$ error rate. Attrition was expected to be $10 \%$. Descriptive statistics are presented as absolute frequency and percentage for categorical variables and mean \pm standard deviation for continuous variables. Associations between the categorical variables were measured in cross-tabulations with chi-square or Fisher's exact tests. Analysis of variance/covariance was used to compare group means and were performed through general linear models. Confidence intervals were adjusted with Bonferroni correction, and effect size $\left(\mathrm{Eta}^{2}\right)$ and observed power were used to evaluate type I and II errors. Mauchly's test of sphericity, with the Greenhouse-Geisse correction for degrees of freedom, was used for repeated measures without assuming equal variance. The frequencies of medication use and nights in which a rescue dose was taken were used as covariates in general linear models for PSG and PVT parameters, controlling for possible residual effects. Intention-to-treat analysis was performed and every randomized participant was included in the analyses. Missing data were replaced by using the last observation carried forward method, in which the last available measurement for each individual prior to discontinuation from the study was retained in the analysis. ${ }^{18}$ Sleep diary data analysis included use of general estimating equations, which do not assume the normality of the dependent measure and allow the selection of different types of distribution probabilities. Since they also allow all recorded daily values to be examined, they were used to evaluate "daily" variations in perceived TST across all treatment phases and to control for possible interaction effects with bedtime, rescue medication, baseline insomnia severity, and age.

\section{Ethics statement}

The study protocol was approved by the Universidade Federal de São Paulo research ethics committee (protocol 184.648), and the study was registered in ClinicalTrials.gov (NCT01896336).

\section{Results}

The mean \pm standard deviation number of days between screening and randomization was $6 \pm 4$. Follow-ups at weeks 2,6 , and 13 occurred $15 \pm 2,31 \pm 4$, and $46 \pm 4$ days after the previous visit, respectively, totaling $92 \pm 5$ days of treatment and $98 \pm 5$ days in the protocol (between March and November 2013).

\section{Study population}

Of the 85 screened individuals, 18 (21\%) were not randomized due to reasons presented in the study flow diagram (Figure 1). The 67 participants were middle-aged (48 \pm 10 years), and most were non-obese women $(n=53$; $79 \%$ ) whose average insomnia duration was $13 \pm 9$ years and who took at least one dose of study medication. All participants attended the week 2 follow-up and were evaluated by clinicians, but not all had completed their sleep diaries and questionnaires or had PSG or PVT measures collected. Participants who completed the protocol $(n=46 ; 69 \%)$ and those who did not had similar baseline characteristics. However, those who did not complete the protocol had more adverse events and fewer signs of improvement. The treatment groups also presented similar baseline characteristics (Table 1).

\section{Adverse events}

Seventeen of the 21 participants who did not complete the protocol reported adverse events (25\% of the total group), seven did not wish to continue (10\%), and 10 were discontinued on medical advice (15\%). Of those who did not wish to continue, three were from the sublingual group and four were from the oral group, mostly women with mild-to-moderate headaches, dizziness and/or gastrointestinal symptoms who had lower compliance rates and concurrent life events. Of the 10 who discontinued based on medical advice, four presented moderate to severe events associated with emotional instability or gastrointestinal symptoms, but recovered within a few days of discontinuation: three of these were in the oral group and one was in the sublingual group. The other six presented persistent mild-to-moderate adverse events or were in need of medication that could interfere with the study's outcomes. The difference in discontinuation frequency between treatment groups was not significant (Figure 1). 


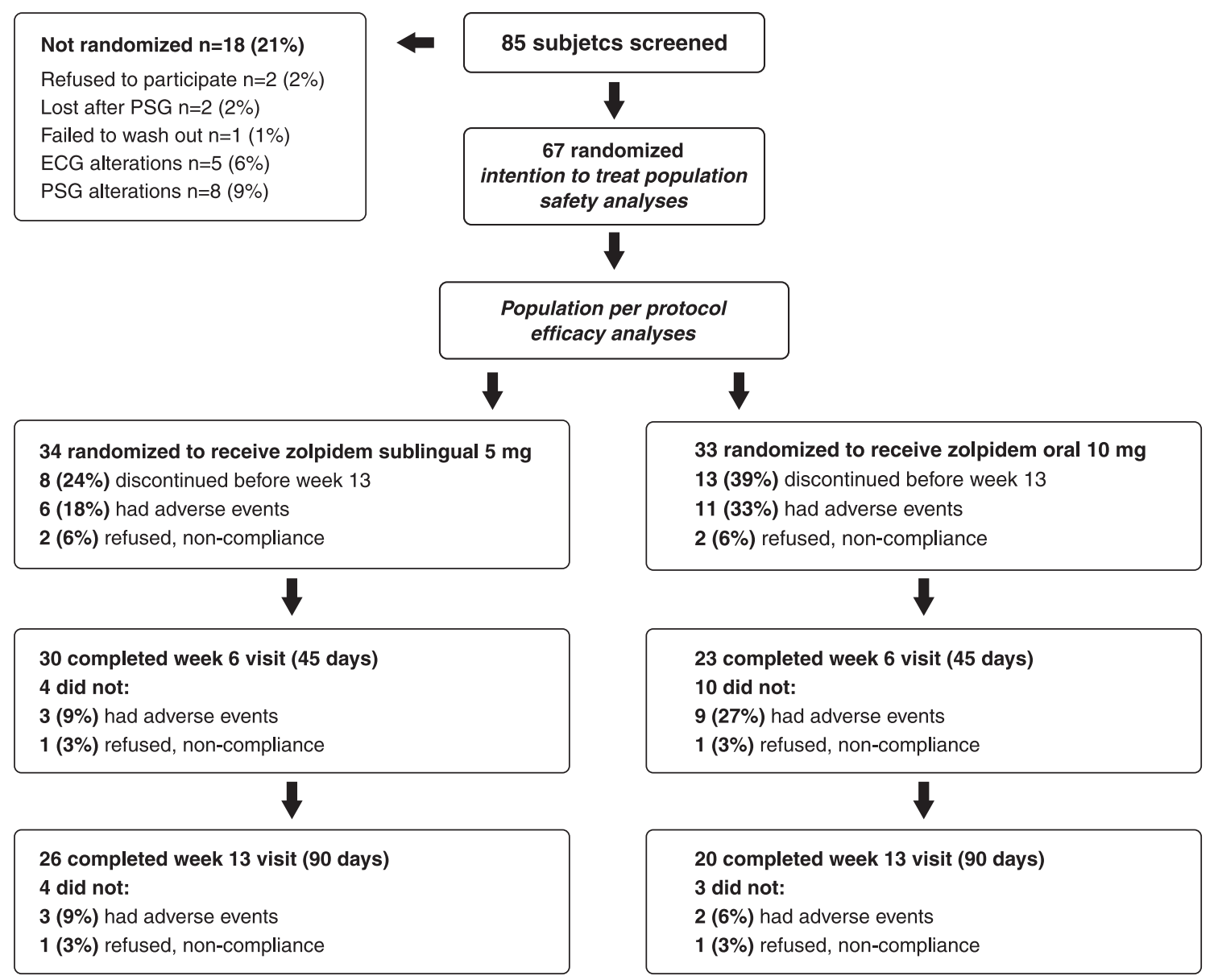

Figure 1 Study flow diagram outlining subject selection and study design. The safety population included all randomized participants who took at least one dose of the study medication. The efficacy population included those who provided any information on treatment effect. Fisher's exact test: no group differences in discontinuation frequency $(24 \mathrm{vs}$. $39 \%$; chi-square = 2.08; two-sided $p=0.18$ ). $E C G=$ electrocardiogram; PSG = polysomnography.

The investigators registered 152 adverse events, 58 (38\%) unrelated to the study medication, $69(45 \%)$ possibly related, and $25(16 \%)$ probably/certainly related. Headache, sleepiness, and dizziness were the most likely events to be treatment-related. The proportion of patients with adverse events did not differ between groups, but the number of treatment-related adverse events leading to discontinuation was higher in the oral group than the sublingual group $(29 \%, n=24$ vs. $13 \%, n=9$; two-sided $p=0.02$ ). See Table 2 for further information.

\section{Efficacy analyses}

Inter- and intragroup sleep diary variance before and after treatment

Both treatments significantly decreased the number of nights per week with MOTN awakenings, on average by $-3.1 \pm 2.3$ days. Before treatment, the sublingual group reported MOTN awakenings an average of $75 \%(95 \% \mathrm{Cl}$ $62-88$ ) of the nights each week, which was reduced to $36 \%(20-53)$ in the third phase of the treatment period. The oral group reported MOTN awakenings an average of
$87 \%(71-100)$ of the nights before treatment, which was reduced to $43 \%(24-62)$ in the third phase of the treatment period. Accordingly, a mean increase of 1.5 hours perceived TST was reported by both groups, with a proportional increase in sleep efficiency (Table 3). Sleeponset latency was the only variable from the sleep diary with an interaction effect and an intergroup difference. There was a greater decrease in sleep-onset latency in the sublingual group than the oral group, even though its baseline values were higher ( 78 vs. $51 ; p=0.03$; effect size $=0.10$; observed power $=0.61)($ Table 3$)$. When comparing group means from the first and second phases of treatment vs. those from the third phase, no intergroup differences were detected in the percentage of nights in which a rescue dose was taken or the number of "as needed" tablets used per week of treatment. Complementary and detailed analyses of sleep diary data are presented in Table 4.

Changes in insomnia severity and sleep quality indexes No significant differences were found in the general linear model between mean questionnaire scores at baseline 
Table 1 Patient sociodemographic and clinical characteristics at baseline

\begin{tabular}{|c|c|c|c|c|}
\hline & $\begin{array}{l}\text { Sublingual zolpidem } \\
\qquad 5 \mathrm{mg}(\mathrm{n}=34)\end{array}$ & $\begin{array}{l}\text { Oral zolpidem } \\
10 \mathrm{mg}(\mathrm{n}=33)\end{array}$ & Total $(n=67)$ & Discontinued $(n=21)$ \\
\hline Age, mean (SD), years & $46(11)$ & $49(10)$ & $48(10)$ & $48(9)$ \\
\hline BMI, mean (SD), $\mathrm{kg} / \mathrm{m}^{2}$ & $26(3)$ & $26(4)$ & $26(4)$ & $26(4)$ \\
\hline Women & $27(79)$ & $26(79)$ & $53(79)$ & $16(76)$ \\
\hline \multicolumn{5}{|l|}{ Marital status } \\
\hline Married & $15(44)$ & $19(58)$ & $34(51)$ & $10(48)$ \\
\hline Single & $11(32)$ & $6(18)$ & $17(25)$ & $5(24)$ \\
\hline Divorced/widowed & $8(24)$ & $8(24)$ & $16(24)$ & $6(28)$ \\
\hline \multicolumn{5}{|l|}{ Education level } \\
\hline Primary and secondary & $22(65)$ & $21(64)$ & $43(64)$ & $12(57)$ \\
\hline Higher & $12(35)$ & $12(36)$ & $24(36)$ & $9(43)$ \\
\hline \multicolumn{5}{|l|}{ Smoking status } \\
\hline Never smoked & $31(91)$ & $30(91)$ & $61(91)$ & $17(81)$ \\
\hline Current or former smoker & $3(9)$ & $3(9)$ & $6(9)$ & $4(19)$ \\
\hline \multicolumn{5}{|l|}{ Clinical history } \\
\hline Treated for insomnia & $13(38)$ & $13(41)$ & $26(39)$ & $8(38)$ \\
\hline Psychoactive drug use & $3(9)$ & $9(27)^{*}$ & $12(18)$ & $4(19)$ \\
\hline Headache & $15(44)$ & $16(49)$ & $31(46)$ & 7 (33) \\
\hline Muscle pain & $10(29)$ & $5(15)$ & $15(22)$ & $5(24)$ \\
\hline Gastrointestinal symptoms & $10(29)$ & $9(27)$ & $19(28)$ & $4(19)$ \\
\hline \multicolumn{5}{|l|}{ Type of symptoms } \\
\hline DIS, DMS or EMA & $14(41)$ & $13(39)$ & $27(40)$ & $10(48)$ \\
\hline Two + symptoms & $20(59)$ & $20(61)$ & $40(60)$ & $11(52)$ \\
\hline $\mathrm{AHI}$, mean (SD) events/hour & $2(2)$ & $4(6)$ & $3(4)$ & $3(3)$ \\
\hline $\mathrm{SaO}_{2} \mathrm{~min}$, mean (SD), \% & $91(3)$ & $90(4)$ & $90(3)$ & $90(3)$ \\
\hline Duration of insomnia, mean (SD), years & $12(9)$ & $15(10)$ & $13(9)$ & $14(8)$ \\
\hline ISI score, mean (SD) ${ }^{\dagger}$ & $17(4)$ & $18(5)$ & $17(5)$ & $18(5)$ \\
\hline PSQI score, mean (SD) ${ }^{\ddagger}$ & $11(3)$ & $12(3)$ & $12(3)$ & $12(3)$ \\
\hline
\end{tabular}

Data presented as $\mathrm{n}(\%)$, unless otherwise specified.

$\mathrm{AHI}=$ apnea and hypopnea index; BMI = body mass index; DIS = difficulty initiating sleep; DMS = difficulty maintaining sleep; EMA = earlymorning awakening; ISI = Insomnia Severity Index; PSQI = Pittsburgh Sleep Quality Index; $\mathrm{SaO}_{2} \mathrm{~min}_{=}$minimum oxyhemoglobin saturation; $\mathrm{SD}=$ standard deviation.

Statistical analyses were performed with two independent binary variables (sublingual zolpidem $5 \mathrm{mg}$ vs. oral zolpidem $10 \mathrm{mg}$, and discontinued protocol vs. completed protocol).

* Statistically significant at $p<0.05$.

$\dagger$ ISI scores range from 0-28 (higher scores indicate more severe insomnia; clinical relevance $>7$ ).

†SQI scores range from 0-21 (higher scores indicate poorer sleep quality; clinical relevance $>5$ ).

Table 2 Number of reported adverse events in association with treatment discontinuation

\begin{tabular}{|c|c|c|c|c|c|c|}
\hline \multirow{2}{*}{ Adverse event types } & \multicolumn{2}{|c|}{ Sublingual zolpidem 5 mg 70 (31) } & \multicolumn{2}{|c|}{ Oral zolpidem 10 mg $82(30)$} & \multicolumn{2}{|c|}{ Total $152(61)$} \\
\hline & Continued & Discontinued & Continued & Discontinued & Continued & Discontinued \\
\hline Central nervous system & $23(16)$ & $7(3)$ & $19(16)$ & $16(7)$ & $42(32)$ & $23(10)$ \\
\hline Respiratory & $15(11)$ & $0(0)$ & $15(11)$ & $0(0)$ & $30(22)$ & $0(0)$ \\
\hline Gastrointestinal & $7(7)$ & $2(1)$ & $14(9)$ & $3(3)$ & $21(16)$ & $5(4)$ \\
\hline Neuromuscular and skeletal & $10(10)$ & $0(0)$ & $4(4)$ & $2(2)$ & $14(14)$ & $2(2)$ \\
\hline Cardiovascular & $2(2)$ & $0(0)$ & $1(1)$ & $1(1)$ & $3(3)$ & $1(1)$ \\
\hline Other & $4(4)$ & $0(0)$ & $5(3)$ & $2(2)$ & $9(7)$ & $2(2)$ \\
\hline Total & $61(27)$ & $9(4)$ & $58(22)$ & $24(8)$ & $119(49)$ & $33(12)$ \\
\hline
\end{tabular}

Data presented as number of events (number of patients).

Continued = continued in the protocol; Discontinued = discontinued participation in the study under medical advice.

In the Total column, the numbers in parentheses may be higher than the total number of participants, since participants could report more than one adverse event during the study.

and the third phase. The mean reduction in Pittsburgh Sleep Quality Index scores was modest but significant, both in the sublingual (mean difference: $-3.4 \pm 4.1$ ) and the oral group (-2.6 \pm 3.4$)$. However, the changes in Insomnia
Severity Index scores were more marked, $-11.3 \pm 5.5$ and $-10.4 \pm 6.1$, respectively (Table 3 ). In the per-protocol analysis, including only individuals who fully completed the trial, the frequency of sleep dissatisfaction according 
Table 3 Inter- and intragroup variance in repeated subjective and objective measures

\begin{tabular}{|c|c|c|c|c|c|c|}
\hline & \multicolumn{2}{|c|}{ Sublingual zolpidem $5 \mathrm{mg}(\mathrm{n}=34)$} & \multicolumn{2}{|c|}{ Oral zolpidem 10 mg (n=33) } & \multicolumn{2}{|c|}{ Within subjects ( $p$-values) } \\
\hline & Baseline & Third phase & Baseline & Third phase & Factor & xGroup \\
\hline \multicolumn{7}{|l|}{ Sleep diaries } \\
\hline Sleep onset latency, minutes & $78(57-99)$ & $25(17-34)$ & $51(27-75)$ & $31(20-41)$ & $<0.001$ & 0.031 \\
\hline Nights with MOTN awakenings, \% & $75(62-88)$ & $36(20-53)$ & $87(71-100)$ & $43(24-62)$ & $<0.001$ & 0.707 \\
\hline Rescue dose nights*, \% & $22(11-33)$ & $15(5-26)$ & $20(7-33)$ & $10(-3-22)$ & 0.223 & 0.799 \\
\hline Total sleep time, hours & $4.8(4.3-5.3)$ & $6.4(5.9-6.9)$ & $4.5(3.9-5.0)$ & $5.7(5.2-6.3)$ & $<0.001$ & 0.390 \\
\hline Sleep efficiency, \% & $62(55-70)$ & $81(75-86)$ & $60(52-69)$ & $76(70-83)$ & $<0.001$ & 0.096 \\
\hline \multicolumn{7}{|l|}{ Questionnaire scores } \\
\hline ISI & $17(15-18)$ & $6(4-8)$ & $18(16-19)$ & $8(6-10)$ & $<0.001$ & 0.470 \\
\hline PSQI & $11(10-12)$ & $8(7-10)$ & $12(11-13)$ & $10(9-11)$ & $<0.001$ & 0.608 \\
\hline KSS, 30 minutes $^{\dagger}$ & $5(4-5)$ & $5(4-6)$ & $5(4-6)$ & $5(5-6)$ & 0.088 & 0.605 \\
\hline KSS, 2 hours ${ }^{\dagger}$ & $4(3-4)$ & $3(3-4)$ & $4(3-4)$ & $4(3-4)$ & 0.178 & 0.233 \\
\hline \multicolumn{7}{|l|}{ PSG parameters } \\
\hline Sleep-onset latency & $27(17-38)$ & $17(9-24)$ & $18(8-28)$ & $24(16-31)$ & 0.502 & 0.031 \\
\hline Wake after sleep onset & $89(39-139)$ & 46 (34-58) & $67(17-118)$ & $52(39-64)$ & 0.102 & 0.440 \\
\hline Total sleep time & $326(306-347)$ & $352(334-369)$ & $333(312-353)$ & $350(332-368)$ & 0.001 & 0.518 \\
\hline Sleep efficiency & $80(75-84)$ & $83(79-87)$ & $80(75-84)$ & $82(78-87)$ & 0.048 & 0.824 \\
\hline Arousal index & $12(10-15)$ & $13(11-16)$ & $16(13-18)$ & $14(12-17)$ & 0.828 & 0.165 \\
\hline REM sleep & $20(18-22)$ & $19(17-20)$ & $19(17-21)$ & $19(17-20)$ & 0.440 & 0.504 \\
\hline Slow wave sleep & $22(19-25)$ & $24(21-27)$ & $22(19-25)$ & $23(21-26)$ & 0.057 & 0.708 \\
\hline $\mathrm{AHI}$ & $2.1(0.6-3.6)$ & $3.1(1.7-4.5)$ & $3.6(2.1-5.1)$ & $3.9(2.5-5.3)$ & 0.183 & 0.448 \\
\hline $\mathrm{SaO}_{2} \mathrm{~min}$ & $91(89-92)$ & $89(88-90)$ & $89(88-91)$ & $88(87-90)$ & 0.002 & 0.591 \\
\hline \multicolumn{7}{|l|}{ PVT parameters $^{\dagger}$} \\
\hline Sleepiness, 30 minutes & $5(4-6)$ & $6(5-7)$ & $5(4-6)$ & $6(5-7)$ & 0.043 & 0.869 \\
\hline Sleepiness, 2 hours & $3(2-4)$ & $3(2-4)$ & $4(4-5)$ & $4(3-5)$ & 0.171 & 0.328 \\
\hline Total hits, 30 minutes & $92(91-94)$ & $90(85-95)$ & $93(92-95)$ & $91(85-96)$ & 0.169 & 0.885 \\
\hline Total hits, 2 hours & $90(86-94)$ & $94(90-98)$ & $95(91-99)$ & $92(88-96)$ & 0.850 & 0.097 \\
\hline Lapses, 30 minutes & $16(10-22)$ & $9(5-12)$ & $5(0-11)$ & $7(3-11)$ & 0.151 & 0.054 \\
\hline Lapses, 2 hours & $7(3-12)$ & $4(2-7)$ & $4(0-9)$ & $4(2-7)$ & 0.279 & 0.363 \\
\hline Reaction time, 30 minutes & $412(359-464)$ & $368(320-416)$ & $340(287-394)$ & 346 (297-395) & 0.285 & 0.162 \\
\hline Reaction time, 2 hours & $336(301-372)$ & $309(273-346)$ & $300(263-336)$ & $313(276-350)$ & 0.625 & 0.151 \\
\hline
\end{tabular}

Data presented as mean (95\% confidence interval), unless otherwise specified.

$95 \% \mathrm{Cl}=95 \%$ confidence interval; $\mathrm{AHI}=$ apnea-and-hypopnea index; ISI = Insomnia Severity Index; KSS = Karolinska Sleepiness Scale;

MOTN awakenings = middle-of-the-night awakenings; PSG = polysomnography; PSQI = Pittsburgh Sleep Quality Index; PVT = Psychomotor Vigilance Test; REM = rapid-eye-movement; $\mathrm{SaO}_{2} \min =$ minimum oxyhemoglobin saturation.

Last observation carried forward (LOCF) = Missing values for discontinued participants were replaced by baseline or the last observed value.

Within-subject statistics are presented for treatment effect alone (Factor) and interaction effect with the treatment group (x Group).

Bold font indicates statistical significance.

* At baseline, rescue dose nights refers to the percentage of nights a rescue dose was taken until the visit at week 6 , i.e. half of the treatment period. It was used as a covariate in the PSG and PVT models to control for residual effects.

Subjective sleepiness and psychomotor performance were measured twice, 30 minutes and 2 hours after awakening from PSG.

Table 4 Means and frequencies of data reported in all sleep diaries

\begin{tabular}{|c|c|c|c|c|c|c|c|c|}
\hline & \multicolumn{4}{|c|}{ Sublingual zolpidem 5 mg } & \multicolumn{4}{|c|}{ Oral zolpidem $10 \mathrm{mg}$} \\
\hline & Basal & Week 2 & Week 6 & Week 13 & Basal & Week 2 & Week 6 & Week 13 \\
\hline Total diaries, $\mathrm{n}$ & 139 & 507 & 912 & 1,203 & 130 & 520 & 745 & 897 \\
\hline Weekends, n (\%) & $48(34)$ & $220(43)$ & $395(43)$ & $510(42)$ & $42(32)$ & $231(44)$ & $324(43)$ & $377(42)$ \\
\hline Nights medication taken & - & 97 & 93 & 96 & - & 84 & 95 & 99 \\
\hline Rescue dose nights & - & 20 & 13 & 13 & - & 14 & 7 & 9 \\
\hline Perceived TST $\geqslant 6$ hours & 33 & $65^{*}$ & $69^{*}$ & $68^{*}$ & 23 & $53^{*}$ & $49 *$ & $54^{*}$ \\
\hline $\mathrm{SOL} \geqslant 30$ minutes & 72 & 39 & $33 *$ & $35^{*}$ & 65 & 41 & $42 *$ & $42 *$ \\
\hline MOTN awakenings & $67 *$ & $52^{\dagger}$ & $41 *$ & $33^{*}$ & $89 *$ & $58^{\dagger}$ & $48^{*}$ & $41 *$ \\
\hline Sleep efficiency $\geqslant 85 \%$ & 37 & $64^{\dagger}$ & $71^{*}$ & $69 *$ & 44 & $57^{\dagger}$ & $59 *$ & $57 \%^{\dagger}$ \\
\hline
\end{tabular}

Data presented as \%, unless otherwise specified.

The chi-square and z-test of column proportions were adjusted for all pairwise comparisons within a row using the Bonferroni correction.

Proportions were compared between treatment groups at each phase of the protocol period.

MOTN awakenings $=$ middle of the night awakening; SOL $=$ sleep-onset latency; $\mathrm{TST}=$ total sleep time

* Statistically significant at $p<0.01$.

${ }^{\dagger}$ Statistically significant at $p<0.05$. 
to the Insomnia Severity Index at week 13 was significantly lower in the sublingual group (16 vs. $43 \%$; chisquare: $p=0.04$ ).

\section{Objective sleep and signs of residual effects}

Findings for the PSG variables were similar to and corroborated those from the sleep diaries. For PSG TST, both groups had a mean increase of 30 minutes from baseline (Table 3). Differences in wake after sleep onset were not significant. As seen by the large standard deviations, individual changes varied consistently, particularly in the sublingual group (mean difference: $-57 \pm 226$ ) and in contrast with the oral group $(-26 \pm 65)$. Sleep-onset latency was the only statistically significant factor: the sublingual group decreased by $-14 \pm 42$ minutes, while the oral group increased by $10 \pm 29$ minutes $(p=0.03)$. The findings were similar after controlling for the percentage of rescue dose nights. Additionally, use of a rescue dose was found to have an interaction effect with changes in TST, sleep efficiency, REM latency, and the respiratory-related variables (Table 3 ).

PVT scores showed minor signs of residual effects. Both groups had a slight increase in sleepiness 30 minutes after awakening, which reversed after 2 hours. A small group difference was observed in the number of lapses after 30 minutes, which also reversed after 2 hours. Sleepiness ratings in the Karolinska Sleepiness Scale corroborated the PVT results (Table 3 ). We further compared PVT variables between patients who had taken $(n=8)$ as needed medication or not $(n=38)$ on the night of PSG and found no differences in sleepiness $(p=0.47)$ or lapses $(p=0.37)$ 30 minutes after awakening.

\section{Intragroup sleep diary variability}

Patients handed in a total of 5,053 sleep diaries: 2,761 (55\%) from the sublingual group, 2,292 (45\%) from the oral group, and 578 (11\%) from participants of both groups who discontinued the treatment protocol. All available data are described below. Of all the reported nights,

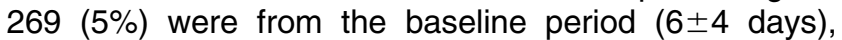
$1,027(20 \%)$ from baseline until the week 2 visit (after $15 \pm 2$ days), 1,657 (33\%) from week 2 to week 6 (after $31 \pm 4$ days), and 2,100 (42\%) from week 6 until the final visit (after $46 \pm 4$ days). Bedtime (11.1 p.m. $\pm 1.2 \mathrm{~h}$ ) and "as needed" (1.9 a.m. $\pm 2.8 \mathrm{~h})$ medication were reported for $96 \%$ and $12 \%$ of the nights, respectively. The total number of nights with reported "as needed" rescue doses were higher in the sublingual group than the oral group ( $n=366$ nights [14\%] vs. $n=193$ nights [9\%]; $p<0.05$ ). Table 4 presents the number of sleep diaries collected at each visit and the valid percentages of complete data reported for each variable, such as the percentage of nights with sleep-onset latency $>30$ minutes $(39 \%)$, MOTN awakenings (45\%), TST > 6 hours (59\%), and ratings of good mood and having had a good night's sleep after awakening (58\%).

Figure 2 compares histograms of sleep diary data throughout the protocol period for both treatment groups, including nightly variations in the number of patients reporting MOTN awakenings or not and whether they took a rescue dose or not. The pattern of rescue dose usage tended to decrease between the first and third phases of treatment in both groups, suggesting no dose escalation or abuse.

According to repeated analysis of the sleep diary data with general estimating equations, controlling for bedtime, the use of rescue doses, age, and baseline insomnia severity, there was no difference between groups in baseline perceived TST. The TST increase in both groups differed somewhat over the treatment phases, there was an interaction effect (Wald $\chi^{2}$ : 94.1; df: $7 ; p<0.001$ ). In the oral group, there was a TST gain of 1.3 hours in the first phase of treatment, which was sustained until week 13. In the

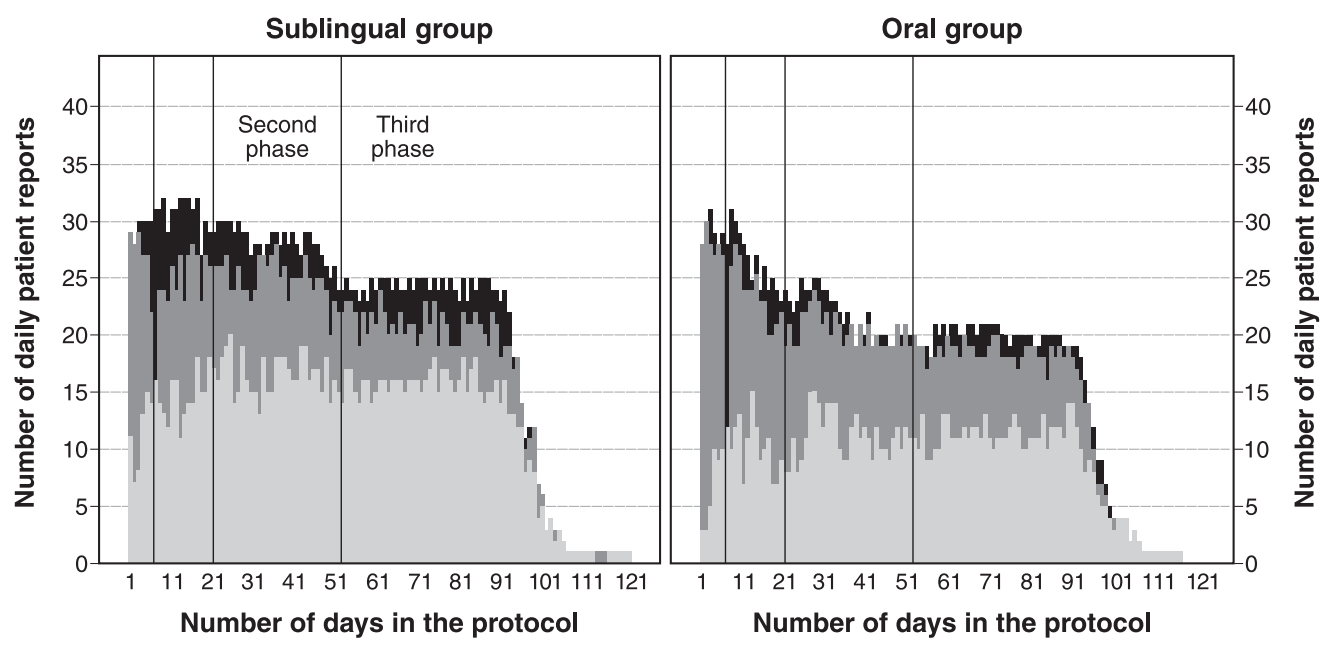

MOTN (+) rescue dose

MOTN (-) rescue dose

No MOTN or rescue dose

Figure 2 Histogram of sleep diary data: number of daily reports during the treatment period stacked according to middle-ofthe-night awakenings with or without the use of "as needed" tablets. MOTN: middle-of-the-night awakenings. 
sublingual group, there was a TST gain of 1.2 hours in the first phase, followed by a mean, progressive, and cumulative increase of 0.2 hour (12 minutes) between the first and second phases, as well as between the second and third phases. MOTN awakenings that led to a rescue dose of medication were associated with a small decrease in perceived TST in the oral group (B: -0.11 ; Wald $\chi^{2}: 11.3$; degrees of freedom: $1 ; p=0.001$ ) but not in the sublingual group (B: -0.01 ; Wald $\chi^{2}$ : 0.1 ; degrees of freedom: 1 ; $\mathrm{p}=0.77$ ). Bedtime (B: -0.03 ; Wald $\chi^{2}$ : 23.6; degrees of freedom: $1 ; p<0.001$ ), but not age or baseline insomnia severity, also exerted a small independent effect on perceived TST.

There was clinical improvement in $37(55 \%)$ participants after completing the protocol: $23(68 \%)$ in the sublingual group and $14(42 \%)$ in the oral group $(p=0.05)$. Most of these participants $(n=23)$ improved after week 6. Additionally, 11 (16\%) participants presented early improvement followed by symptom recurrence, and there was no clinical improvement in $19(28 \%)$ participants. There was no symptom remission in 30 of the randomized participants by the end of the study.

\section{Discussion}

Our main objective was to describe the safety and efficacy of different dosages and treatment schemes of zolpidem in a 3-month trial. Patients from both groups presented no abuse-related behavior and no adverse events severe enough to discourage either drug formulation, which agrees with previous findings. $8,9,19-21$

Both treatments increased TST and sleep efficiency and decreased wake after sleep onset. Regarding sleeponset latency, the sublingual version had faster absorption and distribution rates, reaching higher concentrations and inducing sleep more rapidly. ${ }^{22-26}$ However, an important factor that we failed to control for was the impact of food/ingestion on pharmacokinetics. ${ }^{27}$ Greenblatt et al. showed that taking zolpidem on a full stomach decreases the maximum concentration and increases the chance of residual sedation.

The influence of gender is also significant. ${ }^{8,28-30}$ Our findings suggested a higher dropout rate among men, although we did not have a representative number of men, and thus could not detect whether this was an interaction effect. In women, especially older women, perhaps $10 \mathrm{mg}$ was a high dose, as has been stated by the U.S. Food and Drug Administration. Moreover, a $5 \mathrm{mg}$ dose may not have been effective in men.

Taking rescue doses might have led to small changes in sleep architecture, such as changing REM latency or slow-wave sleep, or to changes in sleep quantity and quality, increasing TST and sleep efficiency. These results are supported by the findings of Roth et al., ${ }^{31}$ who found a dose-dependent effect on the same sleep parameters with even lower doses. These authors reported that improved sleep architecture led to better subjective outcomes, and our findings point in the same direction. It should be mentioned that our use of the last observation carried forward method to deal with missing values or intention-totreat analyses was a conservative measure. It could have underestimated associations, not only with PSG results, but with PVT scores as well. Nevertheless, the participants' performance and sleepiness results did not suggest unmanageable residual effects. Studies using different methods, mostly in healthy individuals, have also found no signs of relevant residual sedation, though patients should always be advised about the risks of activities that require alertness early in the morning. ${ }^{8,21,24,26,31,32}$

Regarding clinical improvement, even though we performed no inter-rater agreement tests between clinicians, they were trained and experienced, as well as blinded. About half of the patients ended treatment with clinically significant symptom remission. Of those whose symptoms recurred, most were in the oral $10 \mathrm{mg}$ group, which was also reflected in sleep dissatisfaction ratings in the questionnaires. The majority of those who had no meaningful improvement had discontinued treatment due to adverse events.

A randomized trial by Morin et al. ${ }^{20}$ evaluated cognitive behavioral therapy (CBT) for insomnia, applied either alone or associated with $10 \mathrm{mg}$ oral zolpidem. After 6 weeks, the CBT-only group remained in the same treatment protocol. Patients in the combined therapy group either continued in the same treatment protocol or switched to CBT alone. After 12 weeks, symptom remission was higher among patients from the combined therapy group that switched to CBT alone than in those who remained in combined therapy (68 vs. $42 \%$ ). We observed the same success rates in our treatment groups: $42 \%$ in the oral group $(n=14)$ and $68 \%$ in the sublingual group $(n=23)$ after the same treatment time, only without CBT. It is possible that the availability of rescue medication gave some patients a sense of control that contributed to lower sleep anxiety and MOTN awakenings. Perlis et al. ${ }^{11}$ suggested something similar in a trial testing intermittent zolpidem doses with and without placebo.

The present study has certain limitations. The heterogeneity and the night-to-night variability in sleep patterns of insomnia patients is hard to address and has been heavily investigated. ${ }^{2,4,33,34}$ Our sample included patients with different durations of insomnia and different histories of psychoactive drug use, and we did not control for possible exposure to previous behavioral interventions, although none of the participants reported previous CBT. Moreover, there was a higher proportion of women, which could increase the probability of slower pharmacokinetics, and we did not control for menopausal status.

It is difficult to recruit patients with untreated insomnia, which contributed to our small sample size. We sought to address this limitation by applying general estimating equation models. Our study is one of the few to have used more robust statistical procedures that can adequately handle a large number of repeated measures, such as the data from the sleep diaries. With general estimating equations, we were able to identify "daily" predictors of changes in perceived TST throughout treatment and the "size" of those changes.

In summary, and in agreement with recommended guidelines, our findings reaffirm that initial lower doses of zolpidem can achieve better overall treatment outcomes while lowering the occurrence of adverse events. 
For some patients, higher dosing regimens may be needed, for others, having rescue medication on hand might be helpful. In a meta-analysis on the effectiveness of non-benzodiazepine GABA-A receptor agonists, HuedoMedina et al. ${ }^{7}$ found that placebo response accounts for, on average, about half of the drug response. Similarly, according to findings by Perlis et al., ${ }^{11}$ alternating nights of drug-therapy and placebo, or stipulating the number of nights per week that a rescue dose can be taken, or other innovative strategies, are potential avenues for clinical and behavioral research and interventions among insomnia sufferers.

In conclusion, both sublingual zolpidem $5 \mathrm{mg}$ (with "as needed" medication) and oral zolpidem $10 \mathrm{mg}$ showed comparable safety and efficacy. Both treatments reduced the number of nights with MOTN awakenings and increased perceived TST. Clinical improvement occurred in $55 \%$ of the participants, of whom $68 \%$ received the sublingual formulation. Sublingual zolpidem was more rapidly absorbed, induced sleep faster, and resulted in a higher frequency of sleep satisfaction.

\section{Acknowledgements}

The authors would like to acknowledge the work of Magda Bignotto, study administrator and leader of the study's monitors and coordinators: Graciela Santos (research analyst), Alexandre Ito (pharmacist), and Merielen CPP Silva (research assistant). The authors would also like to thank Dr. Luciano Rotella and Dr. Erika Tremptow, clinicians involved in patient evaluation and screening, as well as Dr. Altay Alves Lino de Sousa, professor of statistics (psychobiology), for his advice.

\section{Disclosure}

This study was conducted at Centro de Desenvolvimento de Estudos Clínicos Brasil and the Sleep Research Center of the Associação Fundo de Incentivo a Pesquisa (AFIP) and the Universidade Federal de São Paulo, and it was sponsored by EMS Pharmaceutical. EMS had no role in the design or conduct of the study; collection, management, analysis, or interpretation of the data; preparation or approval of the manuscript; or the decision to submit the manuscript for publication. All authors have filled out a disclosure form: LSC has received honorariums and consults for EMS; CFS was a clinician involved in evaluating participants; ST presides over AFIP, which has received a grant from EMS for the present study; DP received a grant from EMS for this study and has received honorariums as a speaker from Sanofi-Aventis and Libbs Pharmaceuticals, for developing and delivering educational presentations and other expenses beyond this study. The authors report no other conflicts of interest.

\section{References}

1 Benca RM. Diagnosis and treatment of chronic insomnia: a review. Psychiatr Serv. 2005;56:332-43.

2 Buysse DJ. Insomnia. JAMA. 2013;309:706-16.

3 American Psychiatric Association. Diagnostic and Statistical Manual of Mental Disorders, Fifth Edition (DSM-5). Arlington: American Psychiatric Publishing; 2013.
4 Vgontzas AN, Fernandez-Mendoza J, Liao D, Bixler EO. Insomnia with objective short sleep duration: the most biologically severe phenotype of the disorder. Sleep Med Rev. 2013;17:241-54.

5 Laugsand LE, Strand LB, Vatten LJ, Janszky I, Bjørngaard JH. Insomnia symptoms and risk for unintentional fatal injuries--the HUNT Study. Sleep. 2014;37:1777-86.

6 Bertisch SM, Herzig SJ, Winkelman JW, Buettner C. National use of prescription medications for insomnia: NHANES 1999-2010. Sleep. 2014;37:343-9

7 Huedo-Medina TB, Kirsch I, Middlemass J, Klonizakis M, Siriwardena AN. Effectiveness of non-benzodiazepine hypnotics in treatment of adult insomnia: meta-analysis of data submitted to the Food and Drug Administration. BMJ. 2012;345:e8343.

8 Greenblatt DJ, Roth T. Zolpidem for insomnia. Expert Opin Pharmacother. 2012;13:879-93.

9 US Food and Drug Administration (FDA). Drug safety communication: risk of next-morning impairment after use of insomnia drugs; FDA requires lower recommended doses for certain drugs containing zolpidem [Internet]. 2013 Oct 1 [cited 2019 Oct 2].

10 Winkler A, Auer C, Doering BK, Rief W. Drug treatment of primary insomnia: a meta-analysis of polysomnographic randomized controlled trials. CNS Drugs. 2014;28:799-816.

11 Perlis M, Grandner M, Zee J, Bremer E, Whinnery J, Barilla H, et al. Durability of treatment response to zolpidem with three different maintenance regimens: a preliminary study. Sleep Med. 2015;16: 1160-8.

12 Buysse DJ, Ancoli-Israel S, Edinger JD, Lichstein KL, Morin CM. Recommendations for a standard research assessment of insomnia. Sleep. 2006;29:1155-73.

13 Buysse DJ, Reynolds CF 3rd, Monk TH, Berman SR, Kupfer DJ. The Pittsburgh sleep quality index: a new instrument for psychiatric practice and research. Psychiatry Res. 1989;28:193-213.

14 Morin CM, Belleville G, Bélanger L, Ivers $H$. The insomnia severity index: psychometric indicators to detect insomnia cases and evaluate treatment response. Sleep. 2011;34:601-8.

15 Kaida K, Takahashi M, Akerstedt T, Nakata A, Otsuka Y, Haratani T, et al. Validation of the Karolinska sleepiness scale against performance and EEG variables. Clin Neurophysiol. 2006;117:1574-81.

16 Iber C, Ancoli-Israel S, Chesson A, Quan S. The AASM manual for the scoring of sleep and associated events: rules, terminology and technical specifications. Westchester: American Academy of Sleep Medicine; 2007

17 Lim J, Dinges DF. Sleep deprivation and vigilant attention. Ann N Y Acad Sci. 2008;1129:305-22.

18 Gupta SK. Intention-to-treat concept: a review. Perspect Clin Res. 2011;2:109-12.

19 Low-dose sublingual zolpidem (Intermezzo) for insomnia due to middle-of-the-night awakening. Med Lett Drugs Ther. 2012;54:25-6.

20 Morin $\mathrm{CM}$, Vallières $\mathrm{A}$, Guay $\mathrm{B}$, Ivers $\mathrm{H}$, Savard $\mathrm{J}$, Mérette $\mathrm{C}$, et al. Cognitive behavioral therapy, singly and combined with medication, for persistent insomnia: a randomized controlled trial. JAMA. 2009; 301:2005-15.

21 Vermeeren A, Vuurman EF, Leufkens TR, Van Leeuwen CJ, Van Oers AC, Laska E, et al. Residual effects of low-dose sublingual zolpidem on highway driving performance the morning after middleof-the-night use. Sleep. 2014;37:489-96.

22 Staner L, Eriksson M, Cornette F, Santoro F, Muscat N, Luthinger R, et al. Sublingual zolpidem is more effective than oral zolpidem in initiating early onset of sleep in the post-nap model of transient insomnia: a polysomnographic study. Sleep Med. 2009;10:616-20.

23 Staner C, Joly F, Jacquot N, Vlasova ID, Nehlin M, Lundqvist T, et al. Sublingual zolpidem in early onset of sleep compared to oral zolpidem: polysomnographic study in patients with primary insomnia. Curr Med Res Opin. 2010;26:1423-31.

24 Roth T, Krystal A, Steinberg FJ, Singh NN, Moline M. Novel sublingual low-dose zolpidem tablet reduces latency to sleep onset following spontaneous middle-of-the-night awakening in insomnia in a randomized, double-blind, placebo-controlled, outpatient study. Sleep. 2013;36:189-96.

25 Valente KD, Hasan R, Tavares SM, Gattaz WF. Lower doses of sublingual Zolpidem are more effective than oral Zolpidem to anticipate sleep onset in healthy volunteers. Sleep Med. 2013;14:20-3.

26 Roth T, Hull SG, Lankford DA, Rosenberg RScharf MB; Intermezzo Study Group. Low-dose sublingual zolpidem tartrate is associated 
with dose-related improvement in sleep onset and duration in insomnia characterized by middle-of-the-night (MOTN) awakenings. Sleep. 2008;31:1277-84.

27 Greenblatt DJ, Harmatz JS, Singh NN, Roth T, Harris SC, Kapil RP. Influence of food on pharmacokinetics of zolpidem from fast dissolving sublingual zolpidem tartrate tablets. J Clin Pharmacol. 2013;53:1194-8.

28 Greenblatt DJ, Harmatz JS, Singh NN, Steinberg F, Roth T, Moline $\mathrm{ML}$, et al. Gender differences in pharmacokinetics and pharmacodynamics of zolpidem following sublingual administration. J Clin Pharmacol. 2014;54:282-90.

29 Roth T, Steinberg F, Singh NN, Moline M. Gender influences on efficacy and safety of sublingual zolpidem tartrate for middle-of-the-night awakening in insomnia. Hum Psychopharmacol. 2014;29:25-30.

30 Walsh JK, Soubrane C, Roth T. Efficacy and safety of zolpidem extended release in elderly primary insomnia patients. Am J Geriatr Psychiatry. 2008;16:44-57.
31 Roth T, Singh N, Steinberg F, Waldron A, Moline M. Sleep architecture following treatment of patients with middle-of-the-night insomnia with buffered sublingual zolpidem compared to placebo. Sleep Med. 2013;14:e211-2.

32 Greenblatt DJ, Harmatz JS, Roth T, Singh NN, Moline ML, Harris $\mathrm{SC}$, et al. Comparison of pharmacokinetic profiles of zolpidem buffered sublingual tablet and zolpidem oral immediate-release tablet: results from a single-center, single-dose, randomized, openlabel crossover study in healthy adults. Clin Ther. 2013;35: 604-11.

33 Buysse DJ, Cheng Y, Germain A, Moul DE, Franzen PL, Fletcher M, et al. Night-to-night sleep variability in older adults with and without chronic insomnia. Sleep Med. 2010;11:56-64.

34 Vallières $\mathrm{A}$, Ivers $\mathrm{H}$, Bastien $\mathrm{CH}$, Beaulieu-Bonneau $\mathrm{S}$, Morin $\mathrm{CM}$. Variability and predictability in sleep patterns of chronic insomniacs. J Sleep Res. 2005;14:447-53. 